AIAA-99-1318

\title{
CONTRIBUTIONS TO ACTIVE BUFFETING ALLEVIATION PROGRAMS BY THE NASA LANGLEY RESEARCH CENTER
}

\author{
Robert W. Moses, AIAA Member \\ Aeroelasticity Branch \\ NASA Langley Research Center \\ Hampton, VA
}

\begin{abstract}
$\underline{\text { Abstract }}$
Buffeting is an aeroelastic phenomenon which plagues high performance aircraft, especially those with twin vertical tails like the F/A-18, at high angles of attack. This buffeting is a concern from fatigue and inspection points of view. By means of wind-tunnel and flight tests, this phenomenon is well studied to the point that buffet loads can be estimated and fatigue life can be increased by structural enhancements to the airframe. In more recent years, buffeting alleviation through active control of smart materials has been highly researched in wind-tunnel proof-of-concept demonstrations and full-scale ground tests using the F/A-18 as a test bed. Because the F/A-18 resides in fleets outside as well as inside the United States, these tests have evolved into international collaborative research activities with Australia and Canada, coordinated by the Air Force Research Laboratory (AFRL) and conducted under the auspices of The Technical Cooperation Program (TTCP). With the recent successes and advances in smart materials, the main focus of these buffeting alleviation tests has also evolved to a new level: utilize the F/A-18 as a prototype to mature smart materials for suppressing vibrations of aerospace structures. The role of the NASA Langley Research Center (LaRC) in these programs is presented.
\end{abstract}

\section{The Problem}

$\underline{\text { Introduction }}$

For high performance aircraft, such as the F/A-18, at high angles of attack, vortices emanating from wing leading edge extensions (LEX) often burst, immersing

Copyright (C) 1999 by the American Institute of Aeronautics and Astronautics, Inc. No copyright is asserted in the United States under Title 17, U. S. Code. The U. S. Government has a royalty-free license to exercise all rights under the copyright claimed herein for Governmental Purposes. All other rights are reserved by the copyright owner. the vertical tails in their wake (Figure 1). Although these vortices increase lift, the resulting buffet loads on the vertical tails are a concern from airframe fatigue and maintenance points of view.

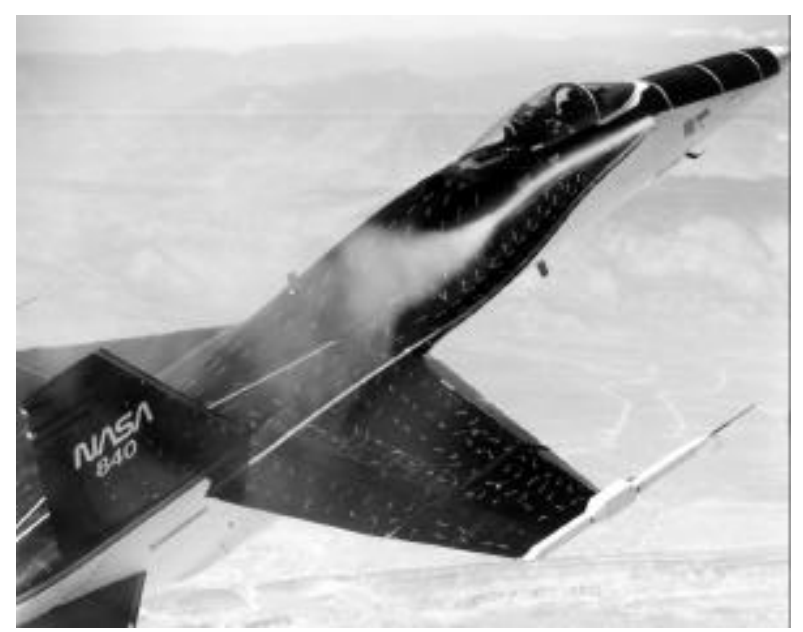

Figure 1. Flow Visualization of Vortex from the LEX Bursting ahead of the Vertical Tail

(Photograph Courtesy of the NASA Dryden Flight Research Center)

\section{$\underline{\text { Previous Investigations }}$}

Previous wind-tunnel and flight tests have been conducted to quantify the buffet loads on the vertical tails of the F/A-18. These tests were designed to characterize the flow mechanism and to quantify the unsteady differential pressures acting on the vertical tails during high-angle-of-attack maneuvers ${ }^{1-7}$. The major findings of these tests were: 1) that the buffet pressures vary with flight conditions; 2) that the buffeting (response of the tail) varies with flight conditions; and 3) that the frequency spectra scale with Strouhal number. Later comparisons among pressure data from reduced-scale wind-tunnel, full-scale windtunnel, and flight tests revealed that the (spatial) correlation time delays as well as the frequency spectra scale with Strouhal number ${ }^{6-7}$. 


\section{$\underline{\text { Past Alleviation Techniques }}$}

Historically, solutions to these fatigue problems have involved controlling the vortex or altering the structural properties of the vertical tail ${ }^{8}$. Vortex control has included blowing and sucking flow from various ports on the aircraft and at various rates ${ }^{9-11}$, attaching different size and shape leading edge extensions (LEX) $)^{12}$, and attaching fences ${ }^{3,4}$ to these LEX. These changes have had limited success since they are effective only at specific flight conditions. Modification of structural properties has included increasing the damping and stiffness of the vertical tail. Structural damping has been increased by applying various concepts such as constrained-layer damping, damped-link, tuned-mass damper, interface damping, and solid-spacer damping ${ }^{13,14}$. Each of these treatments has demonstrated the ability to effect the dynamic response of the structure, but is limited to specific locations, modes, or within specific operating environments.

Stiffness increases have been implemented by means of increasing skin or spar thickness or applying reinforcing members such as brackets, cleats, or doublers. An example of a structural stiffness modification is the composite skin doubler, or "Exoskin," devised by McDonnell Douglas Corporation for a specific series of fighter aircraft ${ }^{15}$. The doubler is bonded to the outboard skin of the vertical tail's main torque box and effectively increases the tail's stiffness. The doubler decreases the load on the internal structure under the patch thus reducing the fatiguing strain in that area. This doubler has effectively eliminated a buffet induced fatigue damage site within those vertical tails with a minimal increase in overall vehicle weight (16 pounds). Often, changes such as these yield the undesirable result of increasing the overall vehicle weight and transfer the load and damage to other structural members. To date, however, the modifications to the set of vertical tails with the doubler attached have not shown counterproductive effects. Ferman et al. ${ }^{15}$ point out that selective stiffening such as this is "especially applicable when a single mode produces a highly concentrated area of strain energy density." However, when the modal strain energy is evenly distributed throughout a structure, alternate means must be sought.

Since passive methods did not fully solve the buffeting problem on the F/A-18, active buffeting alleviation control schemes were investigated. Rock et al. ${ }^{16}$ analyzed active buffeting alleviation systems for the F-
15 and F/A-18 that employed the rudder as the primary actuator. For the F/A-18, their analyses showed that if the rudder actuation frequency bandwidth could be increased to include that of the first two structural modes, then the root mean square (rms) of the root bending moment could be reduced by as much as 33 percent using 3.2 degrees $\mathrm{rms}$ rudder deflection. Furthermore, their analyses showed that the fatigue life might be increased by a factor as high as 25 . Lazarus, Saarmaa, and Agnes ${ }^{17}$ developed an analytical model of an active buffeting alleviation system utilizing linear control algorithms and distributed, layered, piezoelectric strain actuators that were bonded to the surface of the vertical tail. Their analysis indicated that more than a 50 percent reduction in the root mean square (rms) strain at the root of the tail could be achieved at selected flight conditions with a smart material system. Moses ${ }^{18,19}$ and Hauch et al. ${ }^{20}$ separately investigated active buffeting alleviation control systems utilizing piezoelectric strain actuators (among other strategies) on sub-scale, twin-tail, fighter-like aircraft models. Both researchers found that active control systems can significantly reduce buffeting. As indicated in Figure 2, Moses' effort also showed that rudder and piezoelectric actuation are similarly effective up to the worst case buffeting conditions. At angles of attack above the worst case condition, the rudder becomes less effective because of separated flow over the aircraft. However, the piezoelectric actuators, that are not dependent upon the flow characteristics, continue to perform well beyond this point.

\section{$\underline{\text { Recent Research }}$}

Several international programs are performing buffeting alleviation research by means of wind-tunnel, ground, and flight test demonstrations of advanced concepts. The Aeroelasticity Branch at the NASA LaRC is contributing to these ongoing programs by testing scaled F/A-18 models in the Transonic Dynamics Tunnel (TDT) and by participating in conducting full-scale ground tests and in planning flight demonstration tests.

During the ACROBAT (Actively Controlled Response Of Buffet-Affected Tails) program at the LaRC, feedback control to the rudder on the starboard fin and to piezoelectric patches on the port fin were implemented to reduce fin buffeting on a 1/6-scale fullspan F/A-18 model ${ }^{18,19}$. The rms value of the root bending moment was reduced by as much as $20 \%$ using magnitudes well below the physical limits of the actuators. These experimental results agree well with 
the analysis results of Reference 16. Other important findings were that the rudder's effectiveness was limited by degrading flow field conditions due to separated flow at high angles of attack. However, the piezoelectric actuators maintained their effectiveness regardless of flight condition. In addition, not only did the modal response in the first three modes (first bending, first torsion, and second bending) of the tail change with angle of attack, but also the frequency of the first bending mode changed with angle of attack while maintaining a constant tunnel speed. As a result, the control laws developed had to be sufficiently robust to account for these changes in the "plant". Similar plant sensitivities were later corroborated in a separate wind-tunnel investigation ${ }^{21}$. To demonstrate active buffeting alleviation during the ACROBAT test, one time-invariant, fixed-parameter, single-inputsingle-output control law worked well to alleviate the buffeting for all flight conditions. This control law was not optimized for any particular flight condition, and it is thought that its performance would be improved considerably using optimal controller design techniques with "plant" information from all possible flight conditions.

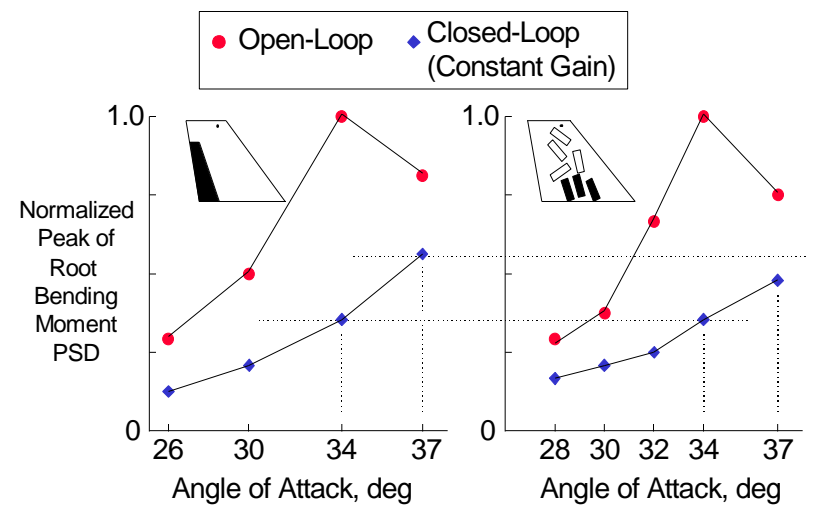
(a) Rudder Results
(b) Piezo Results

Figure 2. Comparisons of the Normalized Peak Values of the PSD of the Root Bending Moment at the Frequency of the First Bending Mode, Open-Loop and Closed-Loop Conditions, Various Angles of Attack, 14 psf

The lessons learned during the ACROBAT program were incorporated into a full-scale ground test of a buffeting alleviation system on an F/A-18 at the AMRL in early 1998. During this AFRL program, conducted under the auspices of TTCP, researchers from the United States, Australia, and Canada used feedback control of piezoelectric patches to reduce the response of the starboard fin to simulated buffet loads. For this test, the International Follow-On Structural Testing Project (IFOSTP) ${ }^{22}$ rig at AMRL (Figure 3) was used. This buffeting alleviation system used off-the-shelf piezoelectric actuators ${ }^{23}$, shown in Figure 4. Researchers from Active Control eXperts (ACX), NASA LaRC, AMRL, and the National Research Institute (NRC) - Canada designed and tested control laws to alleviate buffeting resulting from four different simulated flight conditions ${ }^{24}$. Unfortunately, the nonlinear effects of the load shaker on the modal response of the tail were not considered in the mathematical models of the "plant"; consequently, the actuators did not provide the same level of effectiveness that was predicted using these mathematical models. As a result, the alleviation targets were not quite reached, as shown in Figure 5. Additionally, to maximize control authority for each simulated flight condition, a separate optimal controller was designed and tested by ACX for each of the four flight conditions illustrated in Figure 5. During the worst case flight condition, only one control law lasted the entire duration of the load cycle without overdriving the amplifiers and thus triggering a shutdown switch inside the amplifiers for protecting the system from harmful electrical inputs. This control law was designed for controlling responses in the first bending mode of the tail only and commanded only one of the two amplifier channels driving the piezoelectric actuators. Further details and results may be found in Reference 24 .

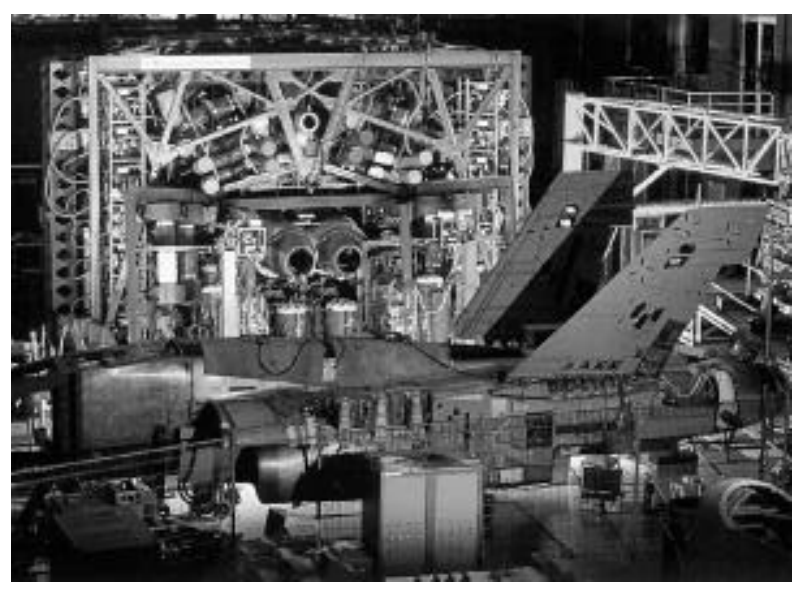

Figure 3. IFOSTP Facility with the F/A-18 Test Article

Although this test was highly successful, recent improvements in amplifiers and piezoelectric patches promise superior capabilities to the system tested at the AMRL. Ongoing programs at AFRL, NASA, and AMRL address the transition of these newly improved components to vibration suppression of aerospace 
structures through wind-tunnel demonstration tests at LaRC, future full-scale ground tests at AMRL, and planned flight demonstration tests at the NASA Dryden Flight Research Center (DFRC).

In a parallel effort at LaRC called SIDEKIC (Scaling Influences Derived from Experimentally-Known Impact of Controls), scaling relationships of piezoelectric actuators are being derived and verified using results from the full-scale ground test at the AMRL and data acquired in the fall of 1998 on a 1/6scaled F/A-18 model tested in the TDT. During this test, additional concepts and configurations were tested as proof-of-concept for buffeting alleviation systems. Additional concepts are planned to be tested in the TDT during 1999. LaRC plans for contributing to these programs are presented next.

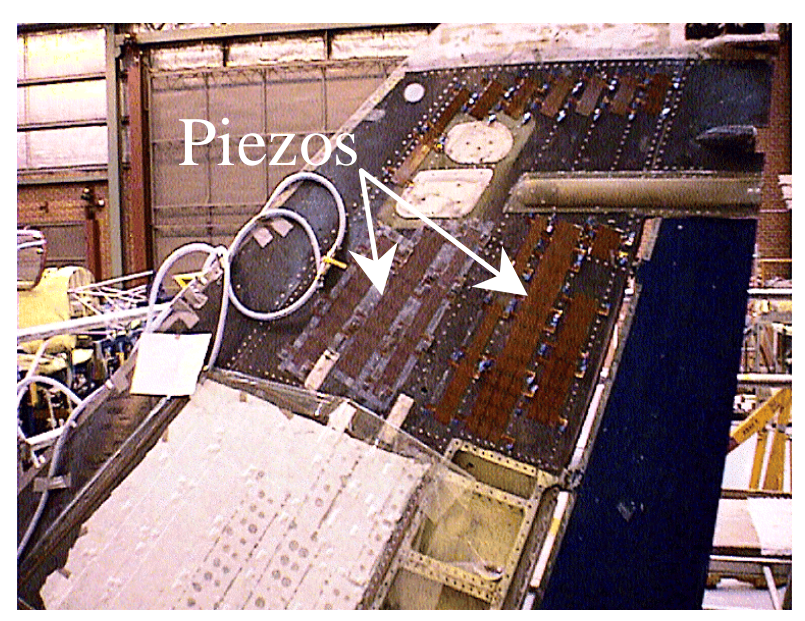

Figure 4. Piezoelectric Actuators Being Bonded to Inboard Surface of the F/A-18 Vertical Tail

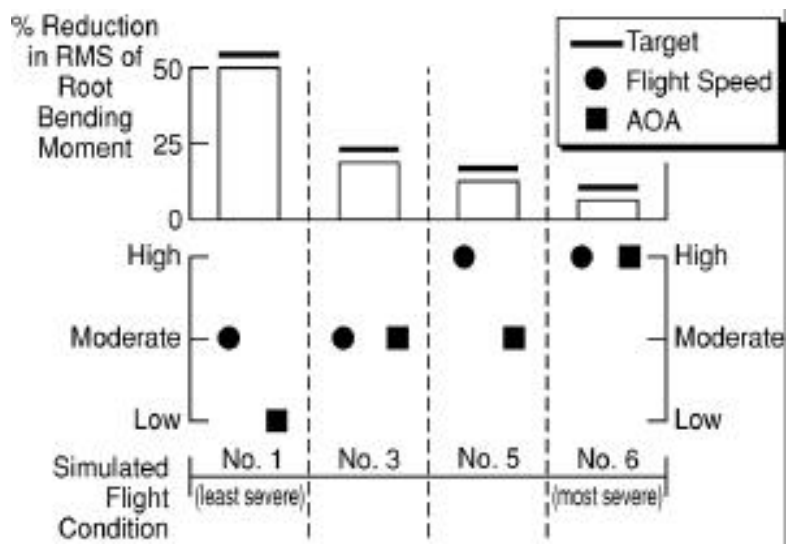

Figure 5. Summary of Alleviation Results for Four Simulated Flight Conditions in the IFOSTP

\section{$\underline{\text { Current Programs and Plans at LaRC }}$}

\section{$\underline{\text { SIDEKIC }}$}

The SIDEKIC model shown in Figure 6 differs from the ACROBAT model in three ways: 1) continuous skin construction was used for the tails; 2) the layout of the piezoelectric actuators; and 3) the type of amplifiers used in the buffeting alleviation system. The port fin, shown in Figure 7, is a scaled version of the buffeting alleviation system tested at the AMRL. After the establishment of target tip deflections and the estimation of force output of the actuators from their specifications, frequency response methods were employed for determining actuator layout and sizes. Results of the ACROBAT and F/A-18 ground test programs were used to guide the establishment of target tip deflections in two ways: 1) the tip deflections caused by the buffeting at various flight conditions were known; and 2) the tip deflections achievable by shaking the tail using just the piezoelectric actuators were known.

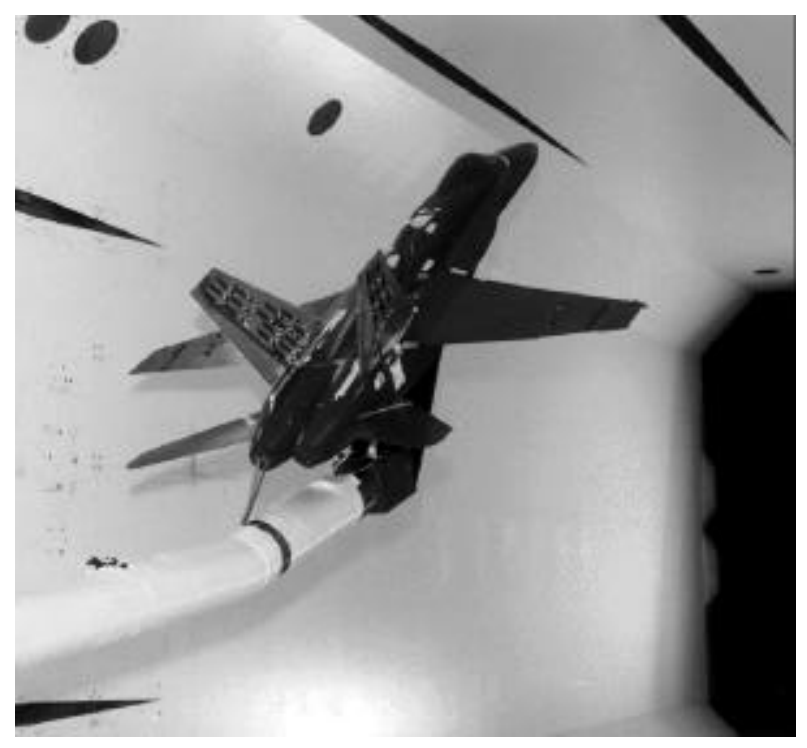

Figure 6. 1/6-scale F/A-18 SIDEKIC Model Mounted in the TDT

For SIDEKIC, a higher tip deflection ratio of item 2 with respect to item 1 was selected as the target because the manufacturer specifications tend to overstate the authority of the actuators that is achievable when bonded to host structure. The resulting additional actuators did not pose a problem for the amplifiers since they could easily handle the additional capacitance load posed by the piezoelectric actuators. Switching amplifiers were selected for the 
SIDEKIC test because they are better suited for driving capacitance loads more efficiently than the linear amplifiers that were used in the ground test. Because the voltage levels required for SIDEKIC were below 200 volts (400 volts peak-to-peak), suitable amplifiers could be obtained from commercial sources.

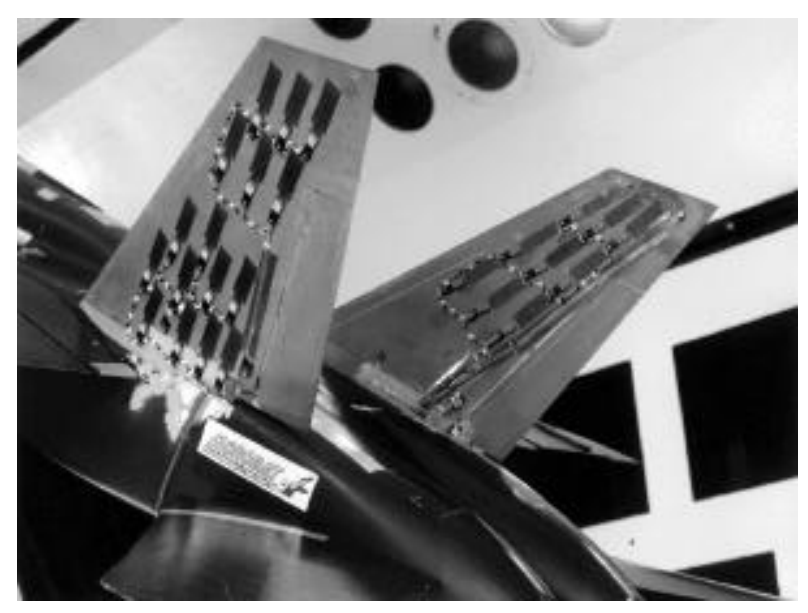

Figure 7. Details of Port Vertical Tail, 1/6-scale F/A18 SIDEKIC Model Mounted in the TDT

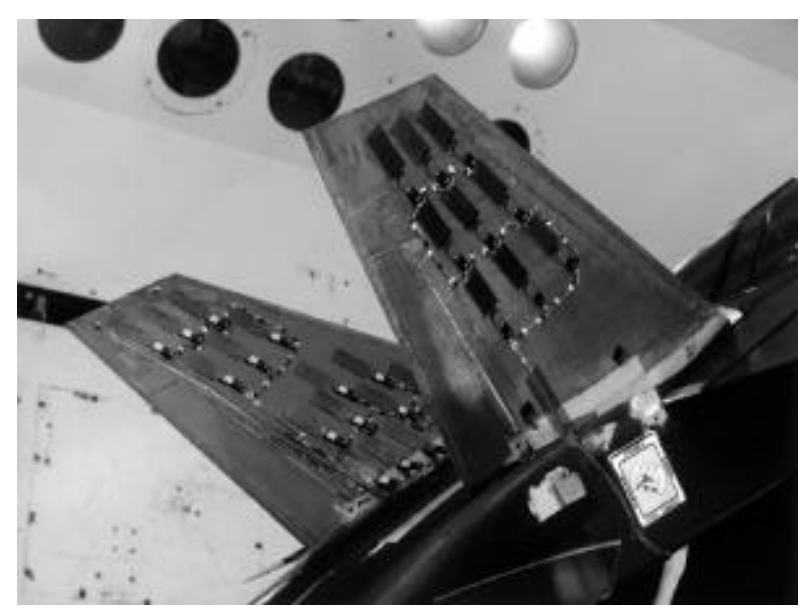

Figure 8. Details of Starboard Vertical Tail, 1/6-scale F/A-18 SIDEKIC Model Mounted in the TDT

The starboard fin, shown in Figure 8, employs an active rudder for controlling responses in the first bending mode, around $16 \mathrm{~Hz}$, and active piezoelectric actuators for controlling responses in the first torsion mode, around $50 \mathrm{~Hz}$. The rudder is activated using a hydraulic actuator and a servo valve. The piezoelectric actuators are the same type used on the port fin. This configuration of control effectors, referred to as a blended system because two actuator technologies were blended, provides a compromise between using an existing control surface and a reduced number (relative to port fin) of additional devices (piezoelectric actuators) that would require integration into an already tightly-packed aircraft.
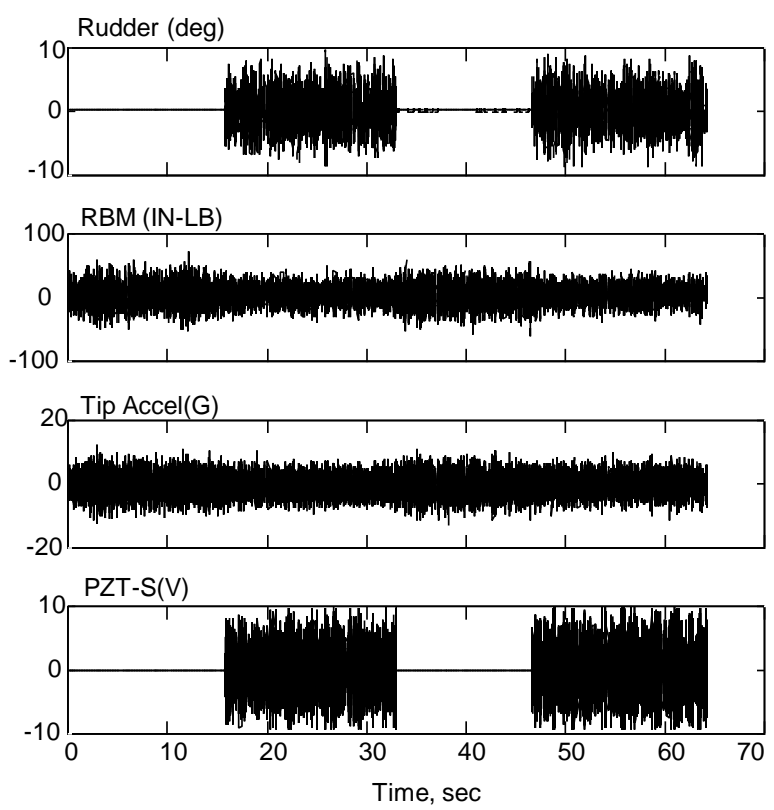

Figure 9. Time Histories of Actuator and Sensors During Feedback Off and On, Blended Buffeting Alleviation System, Starboard Vertical Tail, 1/6-scale F/A-18 SIDEKIC Model

A variety of control schemes were investigated for alleviating the tail buffeting. Boeing, who participated in the SIDEKIC tests through a Memorandum of Agreement (MOA), designed and tested a variety of shunt circuits and neural predictive controllers. A variety of modern state-space controllers were designed by LaRC and tested for reducing tail buffeting in the first two modes mentioned previously. For example, shown in Figure 9, simultaneous feedback control of the rudder (top graph) and of the piezoelectric actuators (bottom graph) caused a pronounced difference in the root bending moment (RBM, second graph) and the tip accelerations (third graph) compared to the open-loop case (when the values of the rudder and piezo commands in top and bottom graphs are zero). For a better comparison, power spectral densities of tip accelerations and root bending moment are computed for open-loop and closed-loop conditions. In Figure 10, significant reductions in the first bending mode (around $17 \mathrm{~Hz}$ ) and the first torsion mode (around $58 \mathrm{~Hz}$ ) were accomplished when feedback to the rudder and piezoelectric actuators are turned on. 


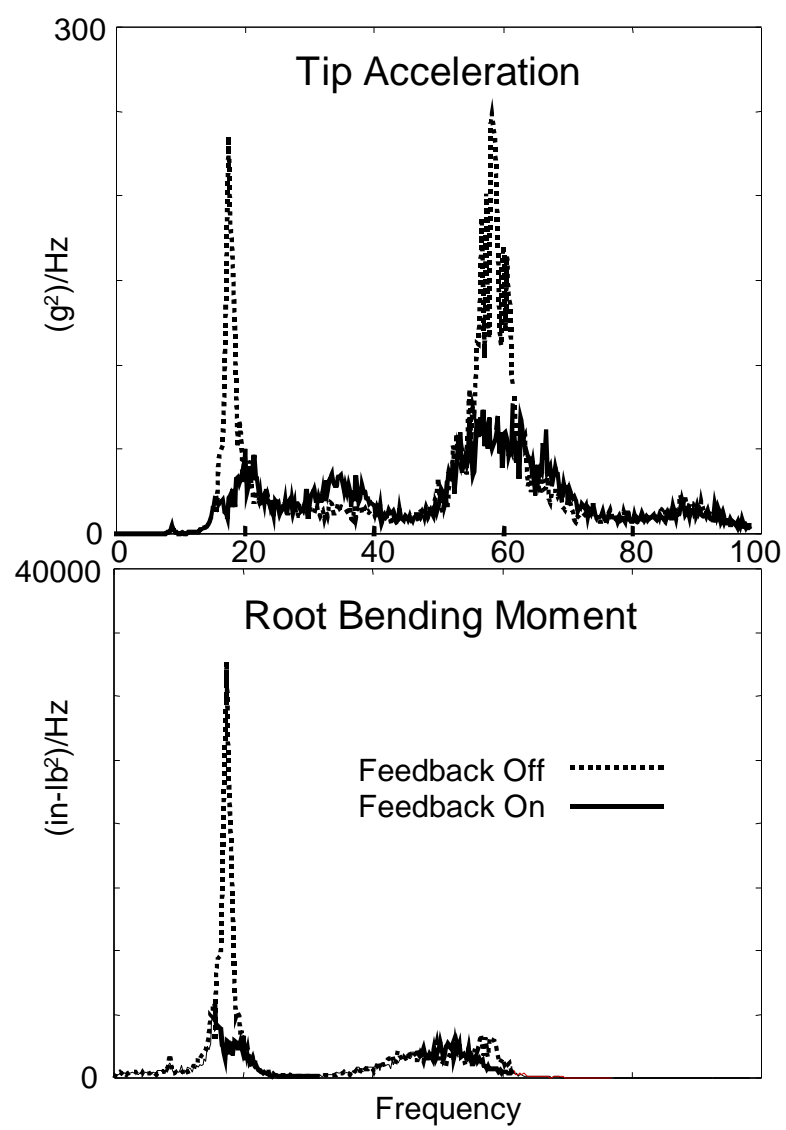

Figure 10. Power Spectral Densities of Buffeting During Feedback Off and On Conditions, Blended Buffeting Alleviation System, Starboard Vertical Tail, 1/6-scale F/A-18 (SIDEKIC) Model

\section{F-22 Buffet Tests}

In collaboration with the F-22 System Program Office (SPO) and the AFRL at Wright-Patterson Air Force Base, a 13.5\% F-22 model (an aerodynamics model designated D6), shown in Figure 11, was refurbished with a new, starboard-side, flexible vertical tail and mounted in the TDT. The purpose of this test was to determine if the F-22 configuration would experience buffeting of the vertical tails similar to that encountered on the F/A-18 at high angles of attack, and to measure the F-22 rudder's effectiveness at the buffeting conditions if encountered. Because of restrictions placed on dissemination of test results by the F-22 SPO, only a description of the test is provided herein.

The flexible tail shown in Figure 12 had a rudder surface driven by a hydraulic actuator for potential buffeting alleviation investigations, and strain gages and accelerometers were bonded to the tail surface for measuring modal response if buffeting was encountered. The rigid, port-side, vertical tail shown in Figure 13 was instrumented with pressure transducers bonded to both surfaces for measuring unsteady pressures. In addition, tests were performed to measure the rudder's effectiveness at high angles of attack. This was accomplished by implementing a closed-loop control system that moved the rudder in response to any buffeting. The control system was designed so that the rudder could reduce buffeting if it occurred. The model was tested at angles of attacks between 7 degrees and 40 degrees and at Mach number between 0.03 and 0.11 .

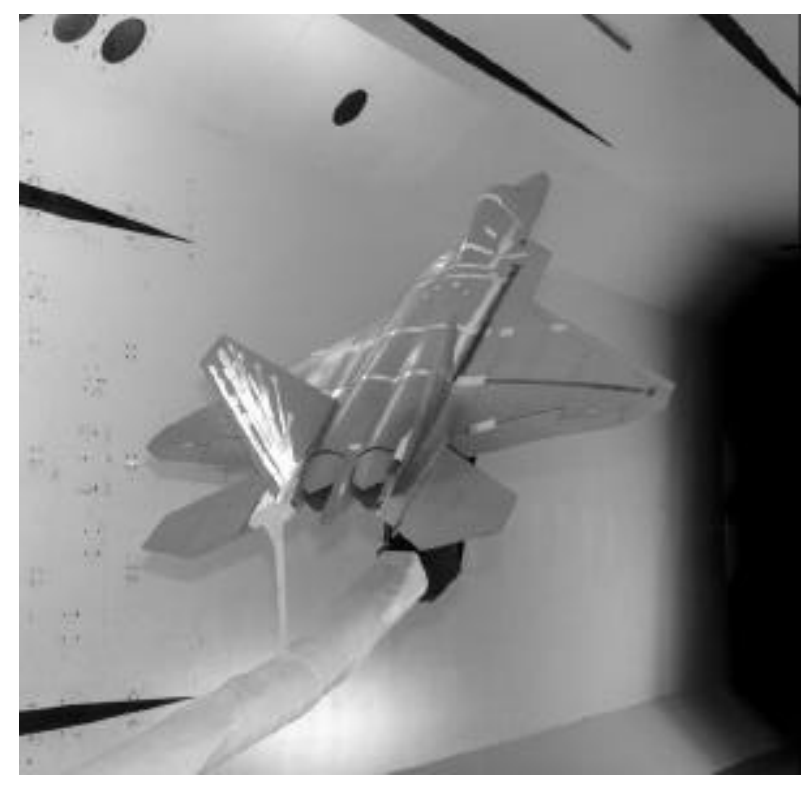

Figure 11. 0.135 Scale F-22 Model Mounted in the TDT

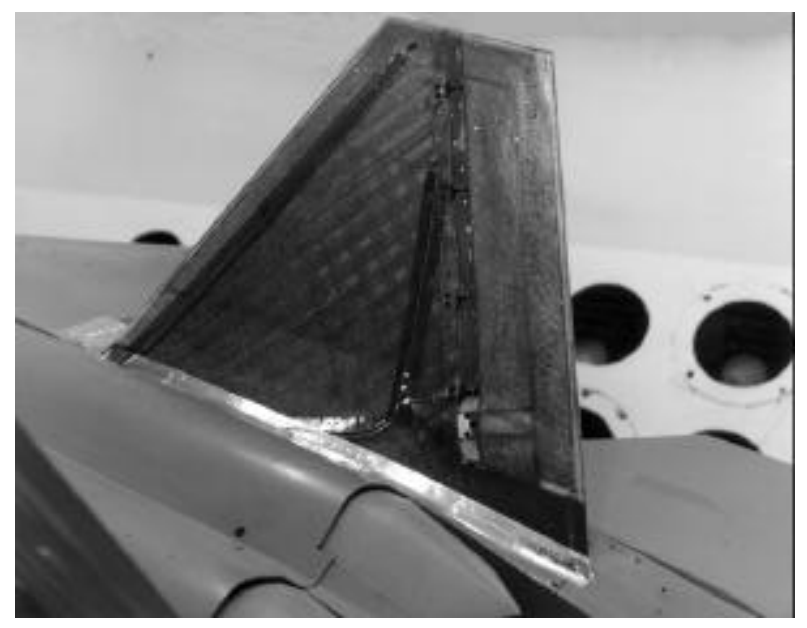

Figure 12. Details of the Flexible Starboard Vertical Tail, 0.135 Scale F-22 Model Mounted in the TDT 


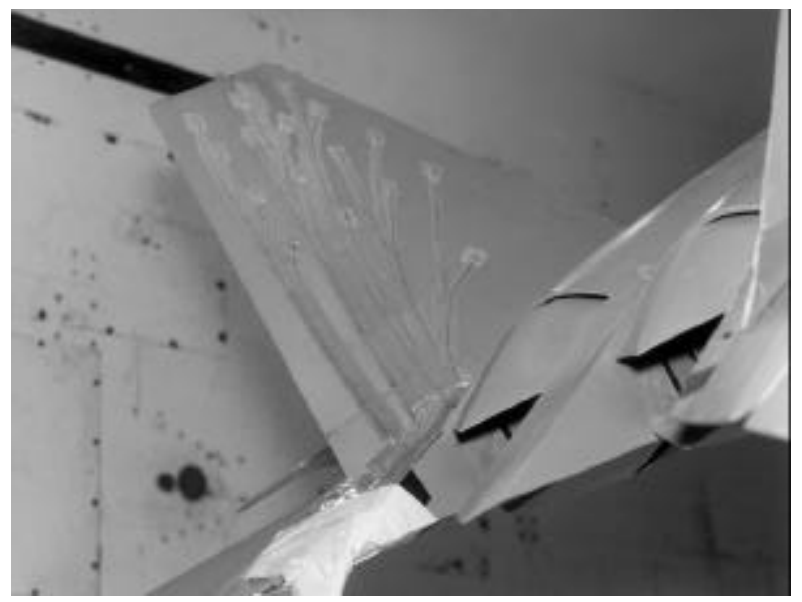

Figure 13. Details of the Rigid Port Vertical Tail, 0.135 Scale F-22 Model Mounted in the TDT

Future Efforts and Their Planned Support by LaRC

\section{Planned Ground Test Follow-On Activity}

Because of the recent significant strides made in piezoelectric actuator and amplifier technologies, the AFRL is planning with the AMRL, NASA (LaRC and DFRC), and the NRC to ground test these new technologies in the IFOSTP rig using the F/A-18 aircraft. The primary actuator candidates are some new interdigitated piezoelectric actuators being developed at LaRC. A program at LaRC is underway to mature these new actuator concepts for ground test program at the AMRL. Within the scope of this program is a planned TDT test of these actuators embedded in the skins of a vertical tail of the 1/6-scale F/A-18 model. This wind-tunnel test will be conducted under the Evaluation of New Actuators in a Buffet Loads Environment (ENABLE) program at LaRC. Following this test, additional lab tests will be conducted for estimating the fatigue life of these actuators when subjected to a high strain environment. Any required enhancements to the actuators will be accomplished prior to the ground test at the AMRL.

\section{$\underline{\text { Planned Flight Tests at NASA DFRC }}$}

Under its Active Buffeting Alleviation of vertical Tails Experiment (ABATE) program, the AFRL plans to flight test a buffeting alleviation system on an F/A-18 at the NASA DFRC. The objective of this program is not only to alleviate buffeting on the F/A-18, but also to demonstrate that the technology has matured sufficiently for vibration suppression of general aerospace structures. In support of this program, LaRC plans to design and test control laws for the rudder and for the piezoelectric actuators, as well as to provide technical oversight as needed. Separate, and as a precursor to the ABATE program, DFRC plans to assess the effectiveness of the rudder on the F/A-18 at high angles of attack for confirming some of the results of the ACROBAT program. LaRC plans to assist DFRC with test planning, some data analysis, and control law design as needed.

\section{$\underline{\text { Conclusions }}$}

The NASA LaRC is playing a vital role in maturing smart materials for vibration suppression on aerospace structures. Specifically, LaRC is conducting windtunnel tests of new devices to demonstrate their effectiveness under specific load conditions. From these tests, LaRC will continue to extend any lessons learned directly to other national and international buffeting alleviation programs. In conjunction with plans at the AFRL to mature smart material technologies for general aerospace use by means of full-scale ground and flight tests, LaRC plans to continue the development of new interdigitated piezoelectric actuators and to use these new devices during future buffeting alleviation demonstrations.

\section{$\underline{\text { Acknowledgments }}$}

The author would like to express his gratitude to the AFRL for its support of the collaborative programs at LaRC in the field of buffeting alleviation. Its support has been critical to the success of the wind-tunnel tests at LaRC. Appreciation is extended to the F-22 System Program Office at Wright-Patterson Air Force Base for loaning the F-22 model for this research. In addition, researchers from ACX, CSA Engineering, the AFRL, the AMRL, and the NRC are acknowledged for their excellent work during the TTCP ground test at the AMRL. It was truly an international collaborative activity that increased the awareness of the thencurrent capabilities of piezoelectric actuators to resolve problems on aerospace structures. The participation of Boeing enhanced the SIDEKIC program significantly. Last, and far from least, sincere gratitude is extended to the LaRC wind-tunnel test teams, and especially the technicians who refurbished many elements of the ACROBAT, SIDEKIC, and F-22 models. The TDT tests could not have been performed without their devotion to this research activity. 


\section{$\underline{\text { References }}$}

1 Zimmerman, N. H., and Ferman, M. A., "Prediction of Tail Buffet Loads for Design Application," Vols. I and II, Rept. No. NADC88043-60, July 1987.

2 Lee, B. H. K., Brown, D., Zgela, M., and Poirel, D., "Wind Tunnel Investigation and Flight Tests of Tail Buffet on the CF-18 Aircraft", AGARDCP-483, Advisory Group for Aerospace Research and Development Specialist's Meeting, Sorrento, Italy, April 1990.

3 Pettit, C. L., Banford, M., Brown, D., and Pendleton, E., "Full-Scale Wind-Tunnel Pressure Measurements on an F/A-18 Tail During Buffet," Journal of Aircraft, Vol. 33, No. 6, NovemberDecember 1996, pp. 1148-1156.

4 Meyn, L. A. and James, K. D., "Full-Scale WindTunnel Studies of F/A-18 Tail Buffet," Journal of Aircraft, Vol. 33, No. 3, May-June 1996, pp. 589595.

5 Moses, R.W. and Ashley, H., "Spatial Characteristics of the Unsteady Differential Pressures on 16\% F/A-18 Vertical Tails," AIAA98-0519, $36^{\text {th }}$ AIAA Aerospace Sciences Meeting and Exhibit, Reno, Nevada, January 12-15, 1998.

6 Moses, R. W. and Shah, G. H., "Spatial Characteristics of F/A-18 Vertical Tail Buffet Pressures Measured in Flight,” AIAA-98-1956, $39^{\text {th }}$ AIAA/ASME/ASCE/AHS/ASC Structures, Structural Dynamics, and Materials Conference and Exhibit, Long Beach, CA, April 20-23, 1998.

7 Moses, R. W. and Pendleton, E., “A Comparison of Pressure Measurements Between a Full-Scale and a 1/6-Scale F/A-18 Twin Tail During Buffet," $83^{\text {rd }}$ Structures and Materials Panel Meeting of the Advisory Group for Aerospace Research and Development (AGARD), 2-6 September 1996, Florence, Italy.

8 Calarese, W. and Turner, E. W., "Progress and New Techniques in Buffet Alleviation," USAF Wright Laboratory Technical Memorandum 973084, September 1997.

9 Bean, D. E., Greenwell, D. I., and Wood, N. J., "Vortex Control Technique for the Attenuation of Fin Buffet," Journal of Aircraft, Volume 30, Number 6, November-December 1993.

10 Mabey, D. G. and Pyne, C. R., "Tangential leading-edge blowing on a combat aircraft configuration," Defence Research Agency Report, 1994.

11 Huttsell, L. J., Tinapple, J. A., and Weyer, R. M., "Investigation of Buffet Load Alleviation on a
Scaled F-15 Twin Tail Model," AGARD SMP Panel Meeting, Aalborg, Denmark, October 1997.

12 Shah, G. H., "Wind-Tunnel Investigation of Aerodynamic and Tail Buffet Characteristics of Leading-Edge Extension Modifications to the F/A18," AIAA Atmospheric Flight Mechanics Conference, AIAA 91-2889, New Orleans, LA, August 12-14, 1991.

13 Gibson, W. C., Maly, J. R., and Austin, E. M., "Conceptual Design of Damping Treatments for the F/A-18 Vertical Tail," CSA Engineering Report No. 88-11-02, November 1988.

14 Liguore, S., Ferman, M., and Yurkovich, R., "Integral Damping Treatment for Primary Aircraft Structures," Damping '91 Conference, San Diego, CA, 13-15 February 1991.

15 Ferman, M. A., Liguore, S. L., Colvin, B. J., and Smith, C. M., "Composite 'Exoskin' Doubler Extends F-15 Vertical Tail Fatigue Life," $34^{\text {th }}$ AIAA Structures, Structural Dynamics, and Materials Conference, La Jolla, CA, 19-22 April 1993.

16 Rock, S. M., Ashley, H., Digumarthi, R., and Chaney, K., "Active Control for Fin Buffet Alleviation," Proceedings from AIAA Guidance, Navigation and Control Conference, 1993, pp. 1051-1056.

17 Lazarus, K. B., Saarmaa, E., and Agnes, G. S., "An Active Smart Material System for Buffet Load Alleviation," Proceedings from SPIE's $2^{\text {nd }}$ Annual International Symposium on Smart Structures and Materials, Volume 2447, 1995, pp. 179-192.

18 Moses, R. W., "Vertical Tail Buffeting Alleviation Using Piezoelectric Actuators - Some Results of the Actively Controlled Response of BuffetAffected Tails (ACROBAT) Program," SPIE's $4^{\text {th }}$ Annual International Symposium on Smart Structures and Materials, San Diego, CA, 4-6 March 1997.

19 Moses, R. W., “Active Vertical Tail Buffeting Alleviation on a Twin-Tail Fighter Configuration in a Wind Tunnel," CEAS International Forum on Aeroelasticity and Structural Dynamics, Rome, Italy, 17-20 June 1997.

20 Hauch, R. M., Jacobs, J. H., Dima, C., and Ravindra, K., "Reduction of Vertical Tail Buffet Response Using Active Control," Journal of Aircraft, Volume 33, Number 3, May-June 1996.

21 Beier, T., Lichtenwalner, P., and Findlay, D.,

"Active Load Alleviation Technology,"

Presentation at the First Annual Joint DoD/NASA/FAA Conference on Aging Aircraft, Ogden, Utah, 10 July 1997. 
22 Conser, D. P., Graham, A. D., Smith, C. J., and Yule, C. L., "The Application of Dynamic Loads to a Full-Scale F/A-18 Fatigue Test Article," $20^{\text {th }}$ Congress of the International Council of the Aeronautical Sciences (ICAS), Sorrento, Napoli, Italy, 8-13 September 1996, ICAS-96-5.10.5, Volume 2, pp. 2465-2480.

23 Moore, J. W., Spangler, R. L., Lazarus, K. B., and Henderson, D. A., "Buffet Load Alleviation Using Distributed Piezoelectric Actuators," Symposium on Adaptive Structures, ASME International Mechanical Engineering Congress and Exposition, Atlanta, GA, November 1996.

24 Hopkins, M., Henderson, D., Moses, R., Ryall, T., Zimcik, D., and Spangler, R., "Active Vibration Suppression Systems Applied to Twin Tail Buffeting," SPIE $5^{\text {th }}$ Annual International Symposium on Smart Structures and Materials, San Diego, CA, 1-5 March 1998. 